\title{
Avaliação da resistência da união imediata à dentina de sistemas adesivos simplificados
}

Immediate bond strength to dentin of simplified adhesive systems

Fernando Freitas Portella1, Vicente Castelo Branco Leitune ${ }^{2}$, Fabrício Mezzomo Collares ${ }^{2}$, Fabrício Aulo Ogliari', Carmen Beatriz Borges Fortes $^{3}$, Susana Maria Werner Samuel ${ }^{3}$

\section{Abstract}

Objetive: The aim of this study was to evaluate the microtensile-bond strength of four commercial adhesive systems. Methods: Twenty bovine incisors had the buccal dentin exposed and restorations were made using four different adhesive systems $(n=5)$ : Magic Bond DE and OptiBond Solo (two-step etch-and-rinse), One-Up Bond F and Adper Prompt L-Pop (one-step self-etch). Teeth were sectioned perpendicularly to the adhesive interface to obtain beams of $1 \mathrm{~mm}^{2}$ of section. The beams were submitted to microtensile-bond strength on a universal testing machine. Data were analyzed by one way ANOVA and Tukey post hoc at a $5 \%$ of significance. Results: The mean bond strength values were $48.81 \pm 0.56$ to Magic Bond DE, $47.52 \pm 13.03$ to OptiBond Solo, 44.68 \pm 13.32 to One-Up Bond $F$ and $31.59 \pm 11.81$ to Adper Prompt L-Pop. Adper Prompt L-Pop exhibited lower bond strength than etch-and-rinse adhesive systems $(p<0.05)$.

Conclusion: Adper Prompt-L-Pop showed the lower immediate bond strength.

Keywords: dentin-bonding agents; dental materials; tensile strength.

\section{Resumo}

Objetivo: Avaliar quatro sistemas adesivos comerciais de diferentes composições quanto ao desempenho da resistência da união à dentina. Métodos: Vinte incisivos bovinos tiveram a dentina vestibular exposta e receberam restaurações de resina confeccionadas com quatro $(n=5)$ diferentes sistemas adesivos simplificados: Magic Bond DE e OptiBond Solo (convencionais), One-Up Bond F e Adper Prompt L-Pop (auto-condicionantes). Os dentes foram seccionados perpendicularmente a interface adesiva utilizando-se um disco de diamante, de modo a se obter palitos de $1 \mathrm{~mm}^{2}$ de área de secção. Os palitos foram submetidos ao ensaio de micro-tração em uma máquina de ensaios universal. Os valores médios de resistência de união dos diferentes sistemas adesivos foram comparados utilizando-se ANOVA de uma via e teste Tukey, em um nível de significância de $5 \%$. Resultados: Os valores médios de resistência da união foram $48,81 \pm 0,56$ para o Magic Bond DE, 47,52 $\pm 13,03$ para o OptiBond Solo, $44,68 \pm 13,32$ para o One-Up Bond $F$ e $31,59 \pm 11,81$ para o Adper Prompt L-Pop. O adesivo Adper Prompt L-Pop apresentou resistência de união inferior a dos adesivos convencionais $(p<0,05)$. Conclusão: Com base no desenho experimental deste estudo, é lícito concluir que o sistema adesivo Adper Prompt-L-Pop apresentou os menores valores de resistência da união adesiva.

Descritores: adesivos dentinários; materiais dentários; resistência à tração.

Rev. Fac. Odontol. Porto Alegre, v. 59, n.1, p. 11-14, jan./jun., 2018.

\begin{abstract}
${ }^{1}$ Cirurgião Dentista, Doutorando em Clínica Odontológica, Laboratório de Materiais Dentários, Faculdade de Odontologia, Universidade Federal do Rio Grande do Sul

2 Doutor em Odontologia, Professor Adjunto de Materiais Dentários, Laboratório de Materiais Dentários, Faculdade de Odontologia, Universidade Federal do Rio Grande do Sul

${ }^{3}$ Doutora em Odontologia, Professora Titular de Materiais Dentários, Laboratório de Materiais Dentários, Faculdade de Odontologia, Universidade Federal do Rio Grande
\end{abstract} do Sul

Correspondência: Fabrício Mezzomo Collares

Laboratório de Materiais Dentários, Faculdade de Odontologia, Universidade Federal do Rio Grande do Sul

Rua Ramiro Barcelos 2492, Porto Alegre, RS, Brasil, CEP 90035-003

Telefone: 555133085198

Email: fabricio.collares@ufrgs.br

\section{Introdução}

A Odontologia adesiva permitiu inúmeros avanços nos procedimentos restauradores, desde a colagem de bráquetes até a cimentação das facetas cerâmicas. A partir do desenvolvimento de materiais e técnicas que permitiram a retenção das restaurações por meio de um embricamento micro-mecânico com a estrutura dentária (BUONOCORE, 1955; NAKABAYASHI et al., 1982), os preparos cavitários passaram a ser mais conservadores, necessitando a remoção de um menor volume de tecido dentário hígido. Entretanto, diversos materiais estão disponíveis no mercado, os quais apresentam diferentes técnicas de aplicação (VAN LANDUYT et al., 2007; OZER e BLATZ, 2013).

Atualmente classificam-se os sistemas adesivos quanto ao condicionamento ácido e número de passos clínicos (VAN LANDUYT et al., 2007). Dentre as variações entre marcas comerciais, os sistemas adesivos apresentam diferenças quanto a sua composição química, como variações da matriz resinosa (BisGMA, UDMA, BisEMA), solvente orgânico (água, álcool, acetona), presença de agente de carga. O desempenho da interface adesiva está diretamente relacionado à composição dos sistemas (PEUMANS et al., 2005). O condicionamento da superfície dentária, de modo que os monômeros possam penetrar na dentina e promover um embricamento micromecânico após a polimerização, pode ser alcançado pela aplicação de um gel de ácido fosfórico previamente a aplicação da resina adesiva, em uma etapa separada, ou pela incorporação de monômeros ácidos na blenda adesiva, de forma que quando aplicada, o condicionamento e a penetração dos monômeros ocorra simultaneamente (VAN MEERBEEK et al., 2011; OZER e BLATZ, 2013).

Frente à diversidade de apresentações comerciais, métodos de aplicação e marcas disponíveis no mercado, é indispensável que os profissionais tenham conhecimento técnico acerca do desempenho dos diferentes sistemas adesivos, para assim tomar a decisão de compra para seus consultórios. Dessa forma, o presente estudo tem como objetivo avaliar quatro sistemas adesivos comerciais de diferentes composições quanto a resistência da união adesiva à dentina. 


\section{Materiais e Métodos}

Vinte incisivos bovinos armazenados em água destilada a $4^{\circ} \mathrm{C}$ após a extração foram utilizados. O esmalte vestibular dos dentes foi desgastado em desgastador de gesso, sob refrigeração, até a exposição de uma área de aproximadamente $100 \mathrm{~mm}^{2}$ de dentina, que então foi polida com lixa d'água de carbeto de silício granulação 600 para que se obtivesse uma smear layer padronizada. Os dentes foram alocados em quatro grupos experimentais $(n=5)$, que receberam restaurações confeccionadas com diferentes sistemas adesivos, aplicados conforme descrito na tabela 1. Foram confeccionadas restaurações cilíndricas de resina (Palfique Estelite, Tokuama Dental Corporation, Japão) de aproximadamente $25 \mathrm{~mm}^{2}$ de área de base e $4 \mathrm{~mm}$ de altura, realizadas em dois incrementos de $2 \mathrm{~mm}$ cada. A fotoativação dos sistemas adesivos e do compósito restaurador foi realizada com aparelho de lâmpada halógena (3M Curing Light XL 2500, 3M, Estados Unidos) com intensidade de luz de $550 \mathrm{~mW} / \mathrm{cm}^{2}$, previamente aferida com radiômetro (Dementron Model 100, Kerr Corporation, Estados Unidos).

Os dentes foram armazenados em água destiladas a $37^{\circ} \mathrm{C}$ por 24 horas. Após esse período, os dentes foram seccionados perpendicularmente a interface adesiva, utilizando-se um disco de diamante em baixa velocidade, sob irrigação constante (Isomet, Buehler Ltd, Estados Unidos), sendo obtidos de 3 a 4 palitos de $1 \mathrm{~mm}^{2}$ de área de secção por dente. Os palitos foram submetidos ao ensaio de micro-tração em uma máquina de ensaios universal (Emic DL-2000, Brasil) equipada com célula de carga de $500 \mathrm{~N}$, a uma velocidade de $1 \mathrm{~mm} / \mathrm{min}$. A resistência de união de cada palito $(\mathrm{MPa})$ foi calculada dividindo-se a carga aplicada $(\mathrm{N})$ pela área adesiva $\left(\mathrm{mm}^{2}\right)$.

Os dados foram submetidos ao teste de Shapiro-Wilk, para verificação da normalidade na distribuição dos dados, e diferenças na média dos valores de resistência da união dos palitos de cada grupo foram determinadas por ANOVA de uma via, seguida do teste de comparações múltiplas de Tukey. Os testes estatísticos foram realizados utilizando-se um nível de significância de 5\%.

\section{Resultados}

A tabela 2 apresenta os valores médios de resistência da união dos sistemas adesivos avaliados. Os valores variaram entre $31,59 \pm 11,81 \mathrm{MPa}$ a $48,81 \pm 10,56 \mathrm{MPa}$. A resistência da união do sistema adesivo Adper Prompt L-Pop foi inferior a dos adesivos convencionais $(p<0,05)$, não havendo diferença entre os valores dos demais adesivos. vantagens desta técnica incluem o controle da hemostasia, mínimo desconforto ao paciente e o fato de dispensar realização de retalho (GÁSPÁR e SZABÓ, 1990; PINHEIRO e FRAME, 1996; CHEE e SASAKI, 2013; LÓPEZ-JORNET e CAMACHOALONSO, 2013). A principal desvantagem do uso de laser de $\mathrm{CO}_{2}$ é o custo do equipamento, necessidade constante de manutenção, bem como necessidade de treinamento e de equipamento de proteção especial para sua utilização. Outra desvantagem é a cauterização das bordas do espécime, dificultando a avaliação das alterações de maturação presentes nestas porções e, consequentemente, a definição de margem livre de alterações (REDDI e SHAFER, 2006; MELTZER, 2007; KHAN et al., 2007).

\section{Discussão}

No presente estudo, os sistemas adesivos testados apresentaram valores de resistência da união comparáveis a estudos prévios (do AMARAL et al., 2010; ULKER et al., 2010; MARCHESI et al., 2013). Buscando diminuir o número de passos clínicos, facilitando a aplicação e diminuindo a sensibilidade técnica, os sistemas adesivos

Tabela 1. Materiais e procedimentos empregados

\begin{tabular}{|c|c|c|}
\hline Sistema adesivo & Classificação & Procedimentos de aplicação \\
\hline $\begin{array}{l}\text { Magic Bond DE } \\
\text { (Vigodent SA, } \\
\text { Brasil) }\end{array}$ & $\begin{array}{l}\text { Convencional } \\
\text { de dois passos }\end{array}$ & $\begin{array}{l}\text { - Condicionamento da dentina com } \\
\text { ácido fosfórico a } 37 \% \text {; } \\
\text { - Lavagem com água destilada por } \\
15 \text { s; } \\
\text { - Secagem com papel absorvente; } \\
\text { - Aplicação vigorosa do adesivo com } \\
\text { aplicador tipo brush por } 15 \mathrm{~s} \text {; } \\
\text { - Evaporação do solvente com jatos } \\
\text { de ar; } \\
\text { - Fotoativação por } 20 \mathrm{~s} \text {. }\end{array}$ \\
\hline $\begin{array}{l}\text { OptiBond Solo } \\
\text { (Kerr } \\
\text { Corporation, } \\
\text { Estados Unidos) }\end{array}$ & $\begin{array}{l}\text { Convencional } \\
\text { de dois passos }\end{array}$ & $\begin{array}{l}\text { - Condicionamento da dentina com } \\
\text { ácido fosfórico a } 37 \% \text {; } \\
\text { - Lavagem com água destilada por } \\
15 \mathrm{~s} \text {; } \\
\text { - Secagem com papel absorvente; } \\
\text { - Aplicação vigorosa do adesivo com } \\
\text { aplicador tipo brush por } 15 \mathrm{~s} \text {; } \\
\text { - Evaporação do solvente com jatos } \\
\text { de ar; } \\
\text { - Fotoativação por } 20 \text { s. }\end{array}$ \\
\hline $\begin{array}{l}\text { One-Up Bond F } \\
\text { (Tokuama } \\
\text { Dental } \\
\text { Corporation, } \\
\text { Japão) }\end{array}$ & $\begin{array}{l}\text { Auto- } \\
\text { condicionante } \\
\text { de um passo }\end{array}$ & $\begin{array}{l}\text { - Mistura de uma gota do frasco A } \\
\text { com uma gota do frasco B; } \\
\text { - Aplicação vigorosa do adesivo com } \\
\text { aplicador tipo brush por } 15 \mathrm{~s} \text {; } \\
\text { - Evaporação do solvente com jatos } \\
\text { de ar; } \\
\text { - Fotoativação por } 20 \mathrm{~s} \text {. }\end{array}$ \\
\hline $\begin{array}{l}\text { Adper Prompt L- } \\
\text { Pop } \\
\text { (3M ESPE, } \\
\text { Estados Unidos) }\end{array}$ & $\begin{array}{l}\text { Auto- } \\
\text { condicionante } \\
\text { de um passo }\end{array}$ & $\begin{array}{l}\text { - Mistura dos componentes } 1 \text { e } 2 \text { do } \\
\text { blister; } \\
\text { - Aplicação vigorosa do adesivo com } \\
\text { aplicador tipo brush por } 15 \mathrm{~s} \text {; } \\
\text { - Evaporação do solvente com jatos } \\
\text { de ar; } \\
\text { - Fotoativação por } 20 \text { s. }\end{array}$ \\
\hline
\end{tabular}

Tabela 2. Resistência de união (média \pm desvio padrão) dos sistemas adesivos avaliados

$\begin{array}{ll}\text { Sistema adesivo } & \text { Resistência de união (MPa) } \\ \text { Magic Bond DE } & 48,81 \pm 10,56^{\mathrm{a}} \\ \text { OptiBond Solo } & 47,52 \pm 13,03^{\mathrm{a}} \\ \text { One-Up Bond F } & 44,68 \pm 13,32^{\mathrm{ab}} \\ \text { Adper Prompt L-Pop } & 31,59 \pm 11,81^{\mathrm{b}}\end{array}$

Letras diferentes representam diferença entre os valores médios de resistência de união (ANOVA, Tukey, $p<0,05$ )

de três passos foram modificados pelos fabricantes. Existem no mercado sistemas adesivos em que monômeros ácidos são combinados ao primer, de forma a dispensar o condicionamento prévio do substrato dentário, denominados adesivos auto-condicionantes, esistemas adesivos em que o primer e a resina adesiva são combinados em um único frasco (VAN LANDUYT et al., 2007). O uso de monômeros de caráter ácido faz com que a hibridização ocorra simultaneamente à infiltração dos monômeros, diminuindo a 
sensibilidade da técnica quando aplicado em dentina, uma vez que sem a necessidade de condicionamento ácido seguido de lavagem, a umidade da dentina não precisa ser controlada. Além disso, o uso de sistemas adesivos auto-condicionantes promove uma adequada infiltração da malha de colágeno desnuda, e assim torna a interface adesiva mais resistente a degradação hidrolítica (CARVALHO et al., 2005). Os sistemas adesivos auto-condicionantes em que o primer e a resina adesiva são aplicados em etapas distintas apresentam desempenho clínico semelhante aos sistemas adesivos convencionais de três passos (PEUMANS et al., 2005).

Contudo, quando os monômeros hidrófilos do primer e os monômeros hidrófobos da resina adesiva são combinados em um único frasco, o desempenho clínico dos procedimentos restauradores é comprometido (PEUMANS et al., 2005). Esse tipo de simplificação leva a uma camada adesiva permeável e de caráter hidrófilo, favorecendo a sua degradação ao longo do tempo (TAY et al., 2002; TAY et al., 2004; VAN MEERBEEK et al., 2005; VAN MEERBEEK et al., 2011). A permeabilidade da matriz polimérica pode aumentar a sorção de água e consequentemente favorecer a oxidação dos polímeros (CHIARAPUTT et al., 2011), os convertendo a moléculas menores que podem ser lixiviadas. Essa degradação afeta as propriedades mecânicas, estabilidade dimensional, biocompatibilidade e compromete a estabilidade da adesão ao longo do tempo (FERRACANE, 2006).

$\mathrm{O}$ adesivo Adper Prompt L-Pop apresentou valores estatisticamente inferiores aos sistemas adesivos convencionais. Uma possível explicação para esse resultado é a composição deste sistema adesivo, que promove um menor $\mathrm{pH}$, chegando a valores inferiores a 1 (CARVALHO et al., 2005). Potencial hidrogeniônico muito baixo em sistemas adesivos autocondicionantes estão relacionados a um desempenho inferior da resistência adesiva (VAN MEERBEEK et al., 2011). Por outro lado, o sistema One-Up Bond $F$, apesar de ser um sistema autocondicionante, apresenta pH 2,6 (FRANCESCANTONIO et al., 2007). Neste estudo, não foi encontrada diferença estatística entre os sistemas adesivos auto-condicionantes. $\mathrm{O} \mathrm{pH}$ inferior do Adper Prompt L-Pop leva a uma dissolução da hidroxiapatita semelhante a obtida pelo condicionamento com ácido fosfórico a 37\% utilizado junto aos sistemas adesivos convencionais. Porém, como ele não requer a lavagem, os fosfatos de cálcio dissolvidos não são removidos e permanecem retidos na interface adesiva, e sendo esses cristais bastante instáveis em meio aquoso, a integridade da camada adesiva é comprometida (VAN MEERBEEK et al., 2011).

Neste estudo a resistência da união foi avaliada imediatamente após a confecção das restaurações, sem que nenhum tipo de simulação do envelhecimento dos espécimes fosse realizado. Um armazenamento em água ao longo do tempo representaria um desafio à integridade da camada adesiva, e os valores de resistência da união poderiam apresentar uma redução (MARCHESI et al., 2013; ZANCHI et al., 2013). A partir das diferenças entre a resistência da união imediata e após o envelhecimento, os sistemas adesivos poderiam ser caracterizados quanto a sua resistência a degradação, possibilitando inferências sobre a longevidade das restaurações confeccionadas com cada um dos sistemas adesivos testados (VAN MEERBEEK et al., 2010). Dessa forma, são necessários estudos que avaliem a resistência da união adesiva após um envelhecimento dos espécimes

\section{Conclusão}

Com base no desenho experimental deste estudo, é lícito concluir que o sistema adesivo Adper Prompt-L-Pop apresentou os menores valores de resistência imediata da união adesiva.

\section{Referências}

BUONOCORE, M. G. A simple method of increasing the adhesion of acrylic filling materials to enamel surfaces. J Dent Res, Chicago, v. 34, n. 6, p. 849-853, Dec. 1955.

CARVALHO, R. M. et al. A challenge to the conventional wisdom that simultaneous etching and resin infiltration always occurs in self-etch adhesives. Biomaterials, Amsterdam, v. 26, n. 9, p. 1035-1042, Mar. 2005.

CHIARAPUTT, S. et al. Biodegradation of all-in-one self-etch adhesive systems at the resin-dentin interface. Dent Mater J, Tokyo, v. 30, n. 6 , p. 814-826, Nov. 2011.

do AMARAL, R. C. et al. Bond strength and quality of the hybrid layer of one-step self-etch adhesives applied with agitation on dentin. Oper Dent, Seatle, v. 35, n. 2, p. 211-219, Mar./Apr. 2010.

FERRACANE, J. L. Hygroscopic and hydrolytic effects in dental polymer networks. Dent Mater, Copenhagen, v. 22, n. 3, p. 211-222, Mar. 2006

FRANCESCANTONIO, M. D. et al. Bond strength evaluation of selfetch and total-etch adhesive systems on intact and ground human enamel. Braz J Oral Sci, Campinas, v. 6, n. 23, p. 1462-1466, Oct./Dec. 2007.

MARCHESI, G. et al. Influence of ageing on self-etch adhesives: onestep vs. two-step systems. Eur J Oral Sci, Copenhagen, v. 121, n. 1, p. 43-49, Feb. 2013.

NAKABAYASHI, N.; KOJIMA, K.; MASUHARA, E. The promotion of adhesion by the infiltration of monomers into tooth substrates. $\mathbf{J}$ Biomed Mater Res, Haboken, v. 16, n. 3, p. 265-273, May 1982.

PEUMANS, M. et al. Clinical effectiveness of contemporary adhesives: a systematic review of current clinical trials. Dent Mater, Copenhagen, v. 21 , n. 9 , p. $864-881$, Sept. 2005.

OZER, F.; BLATZ, M. B. Self-etch and etch-and-rinse adhesive systems in clinical dentistry. Compend Contin Educ Dent, Lawrenceville, v. 34, n. 1, p. 12-18, Jan. 2013.

TAY, F. R. et al. Single-step, self-etch adhesives behave as permeable membranes after polymerization. Part I. Bond strength and morphologic evidence. Am J Dent, San Antonio, v. 17, n. 4, p. 271-278, Aug. 2004.

TAY, F. R. et al. Single-step adhesives are permeable membranes. J Dent, Bristol, v. 30, n. 7-8, p. 371-382, Sept./Nov. 2002.

ULKER, M. et al. Effect of artificial aging regimens on the performance of self-etching adhesives. J Biomed Mater Res B Appl Biomater, Haboken, v. 93, n. 1, p. 175-184, Apr. 2010.

VAN LANDUYT, K. L. et al. Systematic review of the chemical composition of contemporary dental adhesives. Biomaterials, Amsterdam, v. 28, n. 26, p. 3757-3785, Sept. 2007.

VAN MEERBEEK, B. et al. Technique-sensitivity of contemporary adhesives. Dent Mater J, Tokyo, v. 24, n. 1, p. 1-13, Mar. 2005. 
VAN MEERBEEK, B. et al. Relationship between bond-strength tests and clinical outcomes. Dent Mater, Oxford, v. 26, n. 2, p. e100-121,

Fev. 2010.

VAN MEERBEEK, B. et al. State of the art of self-etch adhesives. Dent Mater, Oxford, v. 27, n. 1, p. 17-28, Jan. 2011.

ZANCHI, C. H. et al. Effects of long-term water storage on the microtensile bond strength of five experimental self-etching adhesives based on surfactants rather than HEMA. Clin Oral Investig., Berlin, v. 17, n. 3, p. 833-839, Aug. 2012. 